\title{
Diagnostic Value and Safety of Penicillin Skin Tests in Children with Immediate Penicillin Allergy
}

\author{
Ilknur Kulhas Celik ${ }^{1}$, Irem Turgay Yagmur ${ }^{1}$, Ozge Yilmaz Topal ${ }^{1}$, MUGE TOYRAN ${ }^{1}$, \\ Ersoy Civelek ${ }^{1}$, Betul Karaatmaca ${ }^{1}$, and Emine Misirlioglu ${ }^{1}$ \\ ${ }^{1}$ Ministry of Health Ankara City Hospital
}

June 4, 2020

\begin{abstract}
Background:The first-line method in the diagnosis of patients who describe an immediate reaction after penicillin intake is skin tests(ST)with penicillin reagents. We aimed to determine the safety and diagnostic value of penicillin STs in the diagnosis of immediate reactions to penicillins. Methods:The study included patients with suspected immediate reaction to penicillin who were subjected to STs using a standard penicillin test kit (Diater;Madrid,Spain) and suspected penicillin and drug provocation tests(DPT) with suspected penicillin at our clinic Results:A total of 191 patients(53.9\%males)with a median age of 6.83 years(inter-quartile range:4.2-12)were included in the study. The time from drug intake to the onset of reaction was [?]1 hour in 138(72.3\%)patients and 1 to 6 hours in 53(27.7\%)patients.Penicillin allergy was confirmed by diagnostic tests in 36(18.8\%)of 191 patients.In multivariate logistic regression analysis, history of both urticaria and angioedema(odds ratio[OR]:27,683 95\%confidence interval[CI]:3.143-243.837, $\mathrm{p}=0.003)$ and anaphylaxis (OR:56.246, 95\%CI:6.598-479.489, $\mathrm{p}<0.001)$ were main predictors of penicillin allergy diagnosis. While STs were positive in 23(63.8\%)patients, $13(26.2 \%)$ patients had positive DPT results despite negative ST results. The negative predictive value(NPV)of STs was calculated $92.2 \%(155 / 168)$. None of our patients experienced immediate or delayed systemic/local reactions in relation to the STs. Conclusions:History of urticaria with and anaphylaxis were main predictors of true penicillin allergy in children with suspected immediate reactions.Skin tests with penicillin reagents are safe for use in children. Although STs have a high NPV, DPT is the gold standard for diagnosis. Drug provocation tests should be performed as the final step of the diagnostic evaluation of penicillin allergy in patients with negative STs
\end{abstract}

\section{INTRODUCTION}

One of the most common causes of drug hypersensitivity reactions (DHRs) in children is beta-lactam antibiotics(BLAs) ${ }^{1}$. The prevalence of self-reported amoxicillin allergy is $1.4 \%$ and $1.2 \%$ for other penicillin allergies(PA) in children ${ }^{2}$.However, confirmed cases in suspected penicillin DHRs in children are much lower ${ }^{3,4}$.Patients with suspected antibiotic allergy, usually have longer hospitalization time, are being prescribed more expensive and broad spectrum antibiotics ${ }^{5-7}$. Therefore, patients with suspected DHRs to penicillin should be evaluated in order to confirm true PA.

Immediate reactions(IR) occur within the first hour of administration of the suspected drug but may occur up to 6 hours and are mediated by drug-specific IgE antibodies. Immediate reactions typically involve urticaria, angioedema, rhinitis, conjunctivitis, bronchospasm, gastrointestinal symptoms, anaphylaxis ${ }^{8}$. Nonimmediate DHRs occur more than one hour after the administration of the causative drug and are often mediated by T-cell. They mostly manifest as a maculopapular rash.The guidelines recommend skin testing as the first-line method for diagnosing IRs to BLAs ${ }^{9,10}$.If nonimmediate cutaneous reaction is mild, DPT can be performed without previous $\mathrm{ST}^{1}$. STs for DHRs to BLAs comprise the major and minor determinants of penicillin, penicillin $\mathrm{G}$ and any other suspected BLAs ${ }^{11}$. Skin tests without PPL and MDM may miss BLA allergy diagnosis in $20 \%$ of the cases. However, this concern is still under debate ${ }^{12}$. 
The negative predictive value(NPV) of the STs is high but some reactions have developed following DPT in patients with suspected PA who have negative ST results ${ }^{13-15}$.

The frequency of systemic reactions related to STs with penicillin reagents in children has been reported to vary between 0.3 to $1.2 \%^{16}$.

The aim of this study was to determine the NPV and safety of STs performed by using the penicillin reagents in children with a history of IRs to penicillins.

\section{METHODS}

The study included patients presenting to our pediatric allergy clinic with a history of IRs to penicillins between January, 2013 and December, 2019 who had undergone STs using a standard penicillin test kit(Diater;Madrid,Spain) and suspected penicillin and DPTs with suspected penicillin. Approval was obtained from the ethics committee of our hospital.

\section{Allergy Work-up}

The initial drug allergy workup started with the standardized European Network of Drug Allergy(ENDA) questionnaire $^{17}$.A history detailed history was taken.If the history was consistent with drug hypersensitivity, then STs and DPTs were performed

The reactions mostly manifested as urticaria, angioedema, bronchospasm and anaphylaxis were classified as immediate when they occurred within the first hour of drug administration. Reactions occurring longer than one hour after drug administration were classified as 'non-immediate reactions'. Most of our patients who had non-immediate mild cutaneous reaction (mostly maculopapuler exanthem) and did not undergo ST before DPT and furthermore they were excluded from the study. However STs were performed on some patients who described an IgE-mediated reaction such as urticaria, angioedema, bronchospasm and anaphylaxis, although they had developed a reaction between 1 to 6 hours, after drug administration and these patients were included in the study.

For patients who had a reaction with phenoxymethylpenicillin (pen-V) or benzylpenicillin (pen-G) and negative ST results with benzylpenicilloyl-poly-L-lysine(PPL), minor determinant mixture(MDM) and PenG, we performed a DPT with pen-V or Pen-G. For patients who had a reaction with aminopenicillins or other penicillins and negative ST results with PPL, MDM, Pen-G and aminopenicillins or other penicillins, we performed DPT with suspected aminopenicillin or other penicillins.

Written informed consent was obtained from the parents or legal guardians of patients.

Prick and Intradermal Testing

Patients were subjected to prick ST with the classic penicillin test kit (including PPL and MDM), Pen$\mathrm{G}(10,000 \mathrm{IU} / \mathrm{mL})$, amoxicillin-clavulanate $(20 \mathrm{mg} / \mathrm{mL})$ and the suspected penicillin. If prick STs were negative, the patients were subjected to intradermal test(IDT) with the classic penicillin test kit, pen-G and the suspected penicillin. We used classic penicillin reagents available in the Diater DAP kit (DAP kit; Madrid, Spain). Amoxicillin-clavulanate [intravenous (iv )form] was used for ST until June 2019 as there were no separate preparations of amoxicillin and clavulanate in our country. After June 2019, we used amoxicillin and clavulanate separately (DAP kit; Madrid,Spain). Medications that could affect the STs or DPTs were stopped 1 week before testing ${ }^{12,18}$.

All of the drugs were initially tested on the forearm skin by using the prick method, and reactions were considered positive when a wheal with a diameter larger than that of the negative control was present 20 minutes later. When prick tests yielded negative results, $0.02 \mathrm{~mL}$ of the relevant agent was injected intradermally on the forearm skin. Readings were taken 20 minutes after injections. Results were considered positive when an increase of $3 \mathrm{~mm}$ in the wheal diameter accompanied by erythema was present. Positive controls for prick tests were carried out with histamine at $10 \mathrm{mg} / \mathrm{ml} .0 .9 \% \mathrm{NaCl}$ was used as negative control. 
When a ST yielded a positive result, the patient was considered to be hypersensitive to the tested drug, and the procedure was ceased.

Drug Provocation Test

An incremental DPT protocol was employed. The therapeutic single and daily doses of the drug were calculated according to age and weight of the patient.The ENDA guidelines for DPTs were followed carefully ${ }^{19}$. The DPT involved administering the suspected drug in divided doses(generally 3-4 doses)every 30 minutes until reaching a cumulative dose close to daily dose of the drug. The test was discontinued in the event of any reaction.Drug administration was open. After the last dose had been administered without a reaction, the patient was kept under surveillance for at least 2 hours.

Statistical Analyses

Statistical analyses were performed using SPSSv.22 statistical software package for Windows(IBM,Chicago, IL,USA). Continuous variables were expressed as mean and standard deviation for data with a normal distribution and as median and interquartile range(IQR) for non-normally distributed data. The chi-square test was used to compare nonparametric data; the Mann-WhitneyU test was used for comparisons among non-normally distributed continuous variables and independent samples t-test for normally distributed continuous variables. A value of $\mathrm{p}<0.05$ was considered statistically significant. Logistic regression analysis was performed to determine the predictors of PA confirmation, in univariable analysis values of 0.20 and below were included in the multivariable analysis. The results were given as odds ratio and $95 \%$ confidence interval. A value of $\mathrm{p}<0.05$ was considered statistically significant.

\section{RESULTS}

206 patients (52.6\% males) with suspected PA underwent STs using the penicillin test kit. Fifteen of these patients were subjected to STs with penicillin test kit and also the suspected drug which gave negative results.However, DPT with the suspected drug could not be performed for these patients because their parents have not consented.These patients were excluded from the study.

A total of 191 patients(53.9\% males) with a median age of 6.83 years(IQR:4.2-12) who had completed allergy workup were included in the study. Amoxicillin-clavulanate was the most commonly suspected $\operatorname{drug}(\mathrm{n}=129$, $67.5 \%)$. Suspected drugs are shown in Table 1 . Urticaria was the most commonly presenting symptom(n=89, $46.6 \%)$ (Table2)

The time from drug intake to the onset of reaction was [?]1 hour in 138(72.3\%) patients and 1 to 6 hours in $53(27.7 \%)$ patients. Of the patients, $154(80.6 \%)$ reported a reaction to aminopenicillins, $36(18.9 \%)$ to pen- $\mathrm{V}$ and pen- $\mathrm{G}$ and one patient to piperacillin-tazobactam

Patients Reporting Reactions to Aminopenicillins

Among 191 patients,154(80.6\%) reported a reaction to aminopenicillins.Allergy was confirmed with diagnostic tests in $33(21.4 \%)$ of patients, of whom $21(63.6 \%)$ had positive STs.

127 patients were subjected to STs with PPL + MDM + Pen-G+amoxicillin-clavulanate(iv). While STs were positive in $7(5.4 \%)$ patients, $10(7.8 \%)$ patients had positive DPT despite negative ST results. Eight patients were subjected to standart amoxicillin and clavulanate antigens separately. While STs were positive in one patient, the other patient had positive DPT despite negative ST results.

Skin prick test was positive in $4(19 \%)$ patients:PPL in one patient, Pen-G in one patient and amoxicillinclavulanate in two patients.Seventeen $(81 \%)$ patients were positive on IDT: amoxicillin-clavulanate in five patients, MDM in six patients, PPL in two patients, clavulanate in one patient, Pen-G in two patients and ampicillin-sulbactam in one patient. Drug provocation test was positive despite negative STs in $12(40 \%)$ patients, of whom 10 had positive DPT with amoxicillin-clavulanate and two had positive DPT with pen-V. Only cutaneous symptoms developed in all DPT-positive patients: urticaria in 11 patients and urticaria with angioedema in one patient. 


\section{Patients Reporting Reactions to Pen-V and Pen-G}

There were 36 patients reporting a DHR to pen-V and pen-G and allergy was confirmed in $2(5.5 \%)$ of them. One of them tested positive on IDT with PPL and the other tested positive on DPT with pen-V. One DPT-positive patient developed urticaria during DPT.

Patients Reporting Reactions to Other Penicillins

One patients reported a reaction to piperacillin-tazobactam. This patient tested negative on STs with penicillin determinants but positive on IDT with piperacillin-tazobactam.

The results of diagnostic tests of patients are summarized in Figure1. In the current study, STs were positive in $23(63.8 \%)$ patients out of 36 patients with PA confirmed with diagnostic tests. Among patients with positive STs, PPL and MDM positivity was detected in 10(43.5\%) patients

While STs were positive in 23(63.8\%)patients, 13(26.2\%)patients had positive DPT despite negative ST.The NPV of STs was calculated as $92.2 \%(155 / 168)$.

None of our patients experienced immediate or delayed systemic/ local reactions in relation to STs. Moreover, mild-to-moderate cutaneous symptoms developed in DPT-positive patients and none of them had a serious systemic reaction.

Predictors of PA diagnosis:

Penicillin allergy was confirmed by diagnostic tests in 36 of patients(18.8\%). Patients with confirmed PA were significantly more likely to develop a reaction within the first hour of drug intake, to present urticaria with angioedema and to report anaphylaxis than patients with unconfirmed $\mathrm{PA}(\mathrm{p}<0.05)$ (Table 2). In the univariate logistic regression analysis, history of urticaria with angioedema, only urticaria, anaphylaxis, concomitant allergic disease and having a reaction within first hour of drug intake were associated with confirmation of PA. In the multivariate logistic regression analysis, history of urticaria with angioedema, only urticaria, anaphylaxis and having a reaction within first hour of drug intake were predictors of confirmation of PA(Table $3)$.

\section{DISCUSSION}

In our study, PA was confirmed in 36(18.8\%) patients and 23(63.8\%) of them tested positive on STs. Among patients with positive STs, positivity was detected with PPL and MDM in 13(56.5\%) patients, amoxicillinclavulanate in seven(30.4\%) patients, ampicillin-sulbactam in one(4.3\%) patient, clavulanate in one $(4.3 \%)$ patient and piperacillin-tazobactam in one(4.3\%) patient. The first-line method for diagnosing IRs to $\beta$ lactams is $\mathrm{ST}^{9,10}$. Previous studies suggested that STs without PPL and MDM may miss a diagnosis of allergy in $20 \%$ of the cases ${ }^{12}$. In our study, PPL and MDM positivity was present in 10 (43.5\%) patients with positive STs.

A range of confirmation rates have been reported in studies evaluating children with suspected BLA allergy but the confirmation rate is often low. In addition, there are patients with negative STs but testing positive on DPT and their prevalence also varies between studies ${ }^{3,13,14,20}$. Ponvert et al. confirmed BLA allergy in $50(30.9 \%)$ of 162 children who developed DHRs within the first hour after exposure to BLAs ${ }^{13}$. They did not use PPL and MDM and 43(86\%) of 50 patients tested positive on STs.In our study, the patients who had reactions within the first six hour of drug intake, especially within the first hour, had a ST positivity in $63.8 \%$ of patients whose PA were confirmed by STs. The majority of our patients were diagnosed using STs but not all.Although we used PPL and MDM in the current study, both the rate of confirmed allergies and the rate of ST positivity were lower than those reported by Ponvert et al.

Atanaskovic-Markovic et al. confirmed allergy using STs (including major and minor determinants)or DPT in $682(58.3 \%)$ of 1170 children developing a DHR within first hour after exposure to penicillin and/or cephalosporin. They found a low number of patients with negative STs and positive DPT among patients with confirmed allergy ${ }^{14}$.In our study, the rate confirmation of drug allergy by diagnostic tests was lower and 
the number of patients with positive DPT despite negative STs was higher in comparison to corresponding figures reported by Atanaskovic-Markovic et al.

In one study, Zambonino et al. confirmed allergy in 9(13.6\%) of 66 patients experiencing a DHR within the first hour after a BLA using the same PPL and MDM as in our study and found DPT positivity in 5 of these 9 patients despite negative STs ${ }^{3}$. Compared to Zambonino et al.'s study, a higher rate of confirmed allergies and a higher ST positivity rate in patients with confirmed allergy were found in our study.

Recently, Solensky et al. conducted a study in adult patients with a history suggestive of IgE-mediated PA using a different penicillin kit with major and minor determinants and reported a NPV of $97.7 \%$ for penicillin STs ${ }^{15}$. In present study we found that the NPV of STs as $92.2 \%(155 / 168)$. The NPV of STs is high in studies involving both children and adults. However, some patients can develop a reaction after negative ST and number of these patients is different among studies. Differences in methodologies used for patient selection, test procedures and populational variances may have effected the results.

In comparison to adults, children have a lower prevalence of systemic reactions associated with STs ${ }^{16}$. Ponvert et al. reported that systemic reactions in relation to STs developed in $2.6 \%$ of patients who underwent STs and all of these reactions were mild or moderate ${ }^{13}$. Atanaskovic-Markovic et al. observed no systemic reactions related to ${ }^{14}$. In our study, none of patients developed systemic reactions during STs, which is consistent with the low rate of systemic reactions reported in literature. However, in our study, we could not evaluate all patients with a history of penicillin anaphylaxis because we only performed tests in those who accepted diagnostic tests and this caused data limitation.

Immediate reactions have more confirmation rate than non-immediate reactions with BLAs in children ${ }^{16}$. Ponvert et al. reported that the confirmation rate of BLA allergy was significantly higher in the children reporting IRs than in the children reporting non-immediate reactions. Additionally, they found that the confirmation rate of BLA allergy was higher in the children reporting anaphylaxis ${ }^{13}$. In the present study, we found that history of having both urticaria and angioedema, anaphylaxis and having a reaction within first hour of drug intake were predictors of confirmation of PA. Both Ponvert et al. and our findings show that patients who describe a reaction within first hour of drug intake reaction and history of anaphylaxis have higher confirmation rate

We have evaluated a large pediatric patient group. All of our patients were subjcted with penicillin standart test kit. However, there are some limitations of our study. Our study was a retrospective study and only a small number $(5.7 \%)$ of our patients with suspected amoxicillin-clavulanate or amoxicillin allergy could be tested with standart amoxicillin and clavulanate antigens separately.

In conclusion, PA was not confirmed in $81.2 \%$ of our patients, indicating the importance of allergy assessment to avoid labeling of patients with a suspected history as "allergic to antibiotic". Additionally, although the majority of patients without true PA could be detected with STs prior to DPT. But, the NPV of STs was not $100 \%$. Our findings show that while STs with penicillin determinants are safe for use in children and have a high NPV, DPT remains the gold standard method for the diagnosis of PA.

\section{References}

1. Gomes ER, Brockow K, Kuyucu Saretta F, Mori F, Blanca-Lopez N, et al. Drug hypersensitivity in children: report from the pediatric task force of the EAACI drug allergy interest group. Allergy, 2016;71(2):149- 161.

2. Mirakian R, Leech SC, Krishna MT, Richter AG, Huber PA, Farooque S, et al. Management of allergy to penicillins and other beta-lactams. Clin Exp Allergy 2015; 45:300-327.

3. Zambonino MA, Corzo JL, Muñoz C, Requena G, Ariza A, Mayorga C, et al. Diagnostic evaluation of hypersensitivity reactions to beta-lactam antibiotics in a large population of children. Pediatr Allergy Immunol 2014; 25: 80-7.

4. Erkocoglu M, Kaya A, Civelek E, Ozcan C, Cakir B, Akan A et al. Prevalence of confirmed immediate type drug hypersensitivity reactions among school children. Pediatr Allergy Immunol 2013;24:160- 
167.

5. Li M, Krishna MT, Razaq S, Pillay D. A real-time prospective evaluation of clinical pharmaco-economic impact of diagnostic label of "penicillin allergy" in a UK teaching hospital. J Clin Pathol 2014;67:108892.

6. Chalabianloo F, Berstad A, Schjott J, Riedel B, Irgens A, Florvaag E. Clinical characteristics of patients with drug hypersensitivity in Norway: a single-centre study. Pharmacoepidemiol Drug Saf 2011;20:506-13.

7. Macy E, Contreras R. Health care use and serious infection prevalence associated with penicillin "allergy" in hospitalized patients: a cohort study. J Allergy Clin Immunol 2014;133:790-6.

8. Romano A, Atanaskovic-Markovic M, Barbaud A, Bircher AJ, Brockow K, Caubet JC, et al.Towards a more precise diagnosis of hypersensitivity to betalactams an EAACI position paper Allergy. 2019 Nov 21. doi: 10.1111/all.14122. [Epub ahead of print]

9. Blanca M, Romano A, Torres MJ, Fernandez J, Mayorga C, Rodriguez J, et al. Update on the evaluation of hypersensitivity reactions to beta-lactams. Allergy 2009;64:183-93

10. Solensky R., Khan D.A., Bernstein I.L., Bloomberg G.R., Castells M.C., Mendelson LM, et al: Drug allergy: an updated practice parameter. Ann Allergy Asthma Immunol 2010; 105:59-273

11. Romano A and Caubet JC. Antibiotic Allergies in Children and Adults: From Clinical Symptoms to Skin Testing Diagnosis.J Allergy Clin Immunol Pract. 2014;2(1):3-12

12. Brockow K, Garvey LH, Aberer W, et al. Skin test concentrations for systemically administered drugs - An ENDA/EAACI Drug Allergy Interest Group position paper. Allergy 68:702- 712, 2013

13. Ponvert C, Perrin Y, Bados-Albiero A, Le Bourgeois M, Karila C, Delacourt C, et al. Allergy to betalactam antibiotics in children: results of a 20-year study based on clinical history, skin and challenge tests. Pediatr Allergy Immunol 2011; 22: 411-8.

14. Atanasković-Marković M, Velicković TC, Gavrović-Jankulović M, Vucković O, Nestorović B. Immediate allergic reactions to cephalosporins and penicillins and their cross-reactivity in children. Pediatr Allergy Immunol 2005; 16: 341-7.

15. Solensky R, Jacobs J, Lester M, Lieberman P, McCafferty F, Nilsson T, et al. Penicillin Allergy Evaluation: A Prospective, Multicenter, Open-Label Evaluation of a Comprehensive Penicillin Skin Test Kit. J Allergy Clin Immunol Pract. 2019;7(6):1876-1885.

16. Bergmann M, Caubet JC. Specific Aspects of Drug Hypersensitivity in Children. Curr Pharm Des. 2016;22(45):6832-6851.

17. Demoly P, Kropf R, Bircher A, Pichler WJ. Drug hypersensitivity: questionnaire. EAACI interest group on drug hypersensitivity. Allergy 1999: 54: 999-1003

18. Brockow K, Romano A, Blanca M, Ring J, Pichler W, Demoly P. General considerations for skin test procedures in the diagnosis of drug hypersensitivity. Allergy 2002;57:45-51.

19. Aberer W, Bircher A, Romano A, Blanca M, Campi P, Fernandez J, et al. Drug provocation testing in the diagnosis of drug hypersensitivity reactions: general considerations. Allergy 2003: 58: 854-63.

20. Diaferio L, Chiriac AM, Leoni MC, Castagnoli R, Caimmi S, Miniello VL, et al. Skin tests are important in children with $\beta$-lactam hypersensitivity, but may be reduced in number. Pediatr Allergy Immunol. 2019;30(4):462-468.

Figure Legend:

Figure1. Diagnostic Test Results of All Patients

\section{Hosted file}

table1.docx available at https://authorea.com/users/329938/articles/456812-diagnostic-valueand-safety-of-penicillin-skin-tests-in-children-with-immediate-penicillin-allergy

\section{Hosted file}

table2 Revised.docx available at https://authorea.com/users/329938/articles/456812diagnostic-value-and-safety-of-penicillin-skin-tests-in-children-with-immediate- 
penicillin-allergy

\section{Hosted file}

table3.docx available at https://authorea.com/users/329938/articles/456812-diagnostic-valueand-safety-of-penicillin-skin-tests-in-children-with-immediate-penicillin-allergy

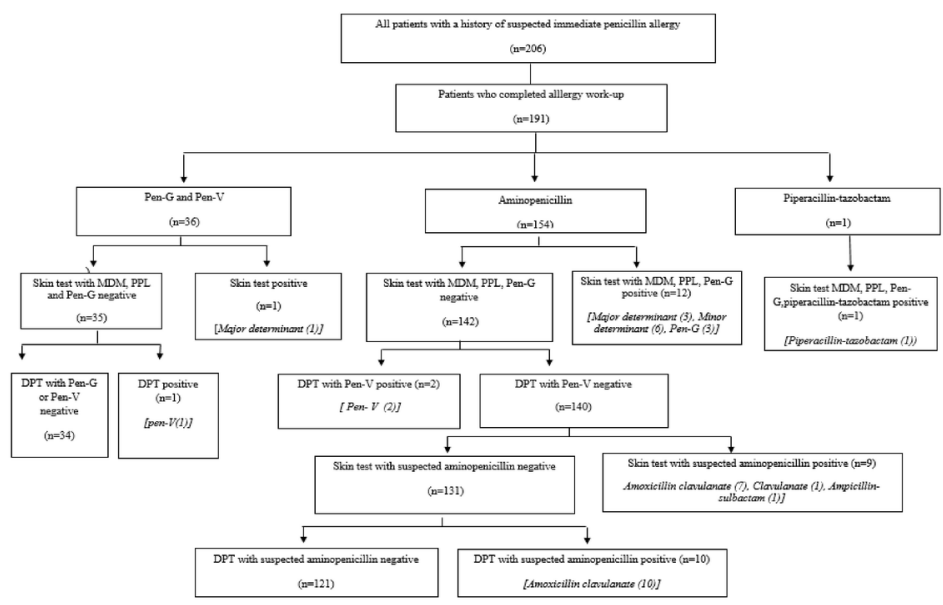

